\title{
Evidências Métricas da Adaptação da Escala de Inteligência Cultural numa Amostra Portuguesa
}

\author{
Metric Evidences of the Adaptation of the Cultural Intelligence Scale \\ in a Portuguese Sample
}

\author{
Cátia Sousa, Gabriela Gonçalves, Marta Reis \& Joana Vieira dos Santos* \\ Universidade do Algarve, Algarve, Portugal
}

\begin{abstract}
Resumo
Este estudo teve como principal objetivo a validação da Cultural Intelligence Scale (CQS) para a população portuguesa. A inteligência cultural é uma competência crucial na adaptação a ambientes multiculturais. A escala multidimensional de inteligência cultural é composta por 20 itens agrupados em quatro dimensões e permite avaliar as características individuais que predispõem um melhor desempenho em ambientes culturais não familiares. Com uma amostra total de 275 participantes, com idades compreendidas entre os 18 e os $72 \operatorname{anos}(M=29,3 ; D P=12,9)$, os resultados obtidos através da realização da análise de componentes principais replicaram os resultados originais, apresentando bons valores de validade e consistência interna (alfa de Cronbach de 0,93), revelando a multidimensionalidade do construto avaliado. A análise exploratória replicou o modelo testado pela análise confirmatória. Assim, a aplicação deste instrumento em contexto organizacional multicultural poderá ser uma ferramenta fundamental para identificar competências cruciais ao desempenho dos recursos humanos, equipas ou organizações, em ambientes caracterizados pela diversidade cultural, assim como, garantir o sucesso dos processos de integração de expatriados e de imigrantes.

Palavras-chave: Inteligência cultural, adaptação portuguesa, Psicometria.
\end{abstract}

\begin{abstract}
Validating the Cultural Intelligence Scale (CQS) for the Portuguese population was the main objective of this study. Cultural Intelligence is recognized as a crucial competence in multicultural situations. The multidimensional cultural intelligence scale is composed of 20 items arranged into four dimensions and is used to evaluate the individual characteristics that predispose to better performance in unfamiliar cultural environments. The results from the analysis of main components in a total sample of 275 participants, aged between 18 and $72(M=29.3 ; S D=12.9)$, replicated the original ones, therefore showing good validity values and internal consistency (Cronbach alpha .93), revealing the multidimension of the evaluated construct. Once the exploratory analysis replicated the model tested by the confirmatory analysis, the application of this instrument in multicultural organizational contexts can be considered an essential tool, not only to identify crucial capacities for the functioning of the human resources, teams or organizations in environments characterized by cultural diversity, but also to ensure the success in the integration processes of immigrants and expatriates.

Keywords: Cultural intelligence, Portuguese adaptation, psychometrics.
\end{abstract}

Para fazer face às constantes mutações que decorrem no mercado global, as organizações e os trabalhadores procuram ajustar-se às variações de oferta e procura, nomeadamente além fronteiras. A procura de melhores condições de vida, o desemprego, a aspiração a uma carreira profissional, entre outros, tendem a aumentar a imigração. Por seu turno, as organizações expandem os seus

\footnotetext{
* Endereço para correspondência: Universidade do Algarve, Faculdade de Ciências Humanas e Sociais, Departamento de Psicologia e Ciências da Educação, Campus de Gambelas, Faro, Portugal 8005-269. E-mail: jcsantos@ualg.pt

Estudo realizado com o apoio do Centro de Investigação sobre Espaço e Organizações (CIEO).
}

negócios para países estrangeiros com vista ao aumento da sua vantagem competitiva, o que implica igualmente um aumento do número de trabalhadores que são destacados para trabalhar no estrangeiro - expatriados.

Contudo a chegada a um novo país, levanta obstáculos e, quer para os expatriados, que vêm apoiados pela empresa-mãe, selecionados através de critérios rigorosos e com formação pré-partida, quer para os imigrantes, que chegam a Portugal sem qualquer apoio organizacional e muitas vezes sem suporte social, o choque cultural é inevitável. Para um ajustamento e integração bem sucedidos, as competências técnicas e profissionais não são suficientes. Cada vez mais os trabalhadores devem estar aptos para lidar diariamente com diferentes contextos culturais (Van 
der Zee \& Van Oudenhoven, 2000). Não basta dominar a língua ou a tarefa que se vai desempenhar. O ajustamento sociocultural está estritamente relacionado com as características individuais e "quanto menos as pessoas estão cientes dos seus próprios enviesamentos culturais, menos competentes serão nas suas relações com os nacionais do país de acolhimento" (Littrell, 2002, citado por Rego \& Cunha, 2009).

A crescente necessidade de internacionalização das organizações e a consequente mobilidade da força de trabalho, obriga muitos trabalhadores a adquirir determinadas competências para lidar com culturas estrangeiras. Do mesmo modo, os gestores devem estar preparados para atuar no campo da diversidade multicultural e liderar equipas multinacionais (Clipa \& Clipa, 2009). A capacidade de interagir eficazmente em múltiplas culturas é uma competência que tem vindo a tornar-se cada vez mais importante no atual mundo laboral e de negócios (Crowne, 2008; Fajardo, 2007), onde se exige cada vez mais trabalhadores aptos para interagir com pessoas de diferentes culturas (Ng \& Earley, 2006), com "capacidade para vencer obstáculos e insucessos" (Rego \& Cunha, 2009 , p. 60). Trabalhar com pessoas de diferentes culturas é um desafio difícil, quer para os trabalhadores, quer para as organizações, devido às barreiras culturais (Ang, Dyne, \& Koh, 2006), uma vez que a cultura engloba um conjunto de valores, crenças, comportamentos, hábitos e atitudes que diferem de sociedade para sociedade (Fan, 2000). A cultura tem um forte impacto nas organizações, já que influencia a comunicação, a liderança, a gestão de equipas, a seleção e formação e, inevitavelmente, a própria cultura organizacional.

Cultura e inteligência são conceitos que estão intrinsecamente interligados, já que a conceptualização, avaliação e desenvolvimento da inteligência não podem ser compreendidos fora do seu contexto cultural, caso contrário a inteligência seria um "construto mitológico" (Sternberg, 2004). Nos últimos anos, têm surgido vários tipos de inteligência (e.g. Gardner, 1983) que realçam a capacidade de adaptação a outros, como a Inteligência Emocional (e.g. Goleman, 1996), a Inteligência Social (Cantor \& Kihlstrom, 1985; Goleman, 2006) ou a Inteligência Interpessoal (e.g. Gardner, 1983). A Inteligência Cultural, sendo compatível com as conceptualizações de Inteligência: capacidade de adaptação e ajustamento ao meio ambiente (Gardner, 1983; Sternberg, 2000), difere dos restantes tipos de inteligência porque se foca especificamente nas interações caracterizadas pela diversidade cultural (Dyne, Ang, \& Koh, 2008). Para Earley e Mosakowski (2004) a inteligência cultural, apesar de estar fortemente ligada à inteligência emocional, ganha onde a inteligência emocional perde. Um indivíduo com uma inteligência emocional elevada abarca o que nos faz humanos e simultaneamente diferentes uns dos outros. Por outro lado, um indivíduo com inteligência cultural elevada é capaz de identificar, a partir do comportamento de um indivíduo ou de um grupo, determinadas características distintivas, sejam elas inerentes a todas as pessoas e a todos os grupos, apenas específicas de uma pessoa ou de um grupo e ainda, características que nem são universais nem idiossincrásicas. $O$ vasto território que jaz entre estes dois pólos é a cultura (Earley \& Mosakowski, 2004).

Para explicar o porquê de alguns indivíduos apresentarem um desempenho mais eficaz que outros em situações multiculturais, Earley e Ang (2003) partiram da perspetiva multidimensional de inteligência de Sternberg e Detterman (1986), para desenvolver um modelo conceptual de inteligência cultural (Rockstuhl, Hong, Ng, Ang, \& Chiu, 2010). A inteligência cultural é motivada pela realidade prática da globalização no local de trabalho (Earley \& Ang, 2003) e é definida como a capacidade de adaptação eficaz em diferentes ambientes culturais (Earley, 2002; Earley \& Ang, 2003), a capacidade de se adaptar a outros (Ng \& Earley, 2006) e a diversas situações culturais (Ang et al., 2006; Earley \& Ang, 2003). É um conjunto de capacidades e competências que permite interpretar comportamentos e situações que não são familiares, bem como identificar comportamentos que são universais a toda a humanidade, comportamentos que são culturais e comportamentos que são idiossincráticos a um determinado indivíduo numa situação especifica (Dyne, Ang, \& Livermore, 2010). À semelhança de Sternberg (1988), para Earley e Ang (2003) a inteligência cultural é um construto multidimensional que compreende 4 dimensões: metacognitiva, cognitiva, motivacional e comportamental. A dimensão metacognitiva corresponde à consciencialização e sensibilização cultural durante a interação com diferentes culturas, sendo uma componente crucial já que promove um pensamento ativo sobre as pessoas e situações num ambiente não familiar. Por outro lado, desencadeia um pensamento crítico sobre hábitos e crenças, assim como permite fazer uma avaliação e revisão dos mapas mentais aumentando assim a capacidade de compreensão (Dyne et al., 2008), ou seja, quem estiver um pouco "desenraizado" da sua própria cultura, poderá mais facilmente adotar os hábitos e costumes, e até mesmo a linguagem corporal, de uma cultura desconhecida (Earley \& Mosakowski, 2004). A dimensão cognitiva refere-se ao conhecimento cultural de normas, comportamentos, práticas e convenções em diferentes culturas, obtido através da experiência e educação, e engloba o conhecimento do sistema económico, social e legal de diferentes culturas e subculturas bem como o conhecimento dos valores culturais (Rose, Ramalu, Uli, \& Kumar, 2010). A dimensão motivacional conceptualiza a capacidade de direcionar a atenção e energia em relação a diferenças culturais, ou seja, é uma forma de autoeficiência e motivação intrínseca em situações interculturais (Dyne et al., 2008). Por exemplo, a inteligência cultural pode facilitar a gestão de stress provocada por contextos multiculturais no trabalho, dotando os indivíduos de confiança para ultrapassar obstáculos. A dimensão comportamental é uma das dimensões mais visíveis da interação social, e refere-se à capacidade para exprimir, verbal e não verbalmente, comportamentos apropriados aquando 
da interação com pessoas de diferentes culturas (Dyne et al., 2008). Ou seja, não se poderá convencer os anfitriões estrangeiros mostrando apenas que se compreende a sua cultura, é necessário prová-lo, através das atitudes, que se está efetivamente integrado no mundo deles (Earley \& Mosakowski, 2004).

No desenvolvimento e validação da escala de inteligência cultural, Dyne et al., (2008) realizaram um primeiro estudo, com uma amostra de 576 participantes. Inicialmente composta por 40 itens, a versão final incluiu apenas 20 , (já que os restantes itens apresentavam altos níveis residuais, pequenos desvios padrões e fraca correlação), que apresentaram fortes propriedades psicométricas da inteligência cultural, e a análise fatorial confirmatória demonstrou um bom ajustamento das quatro dimensões do modelo. $\mathrm{O}$ segundo estudo (generalização por amostras) foi efetuado com uma amostra distinta composta por 447 participantes e revelou igualmente uma forte relação entre os itens e as 4 dimensões. O terceiro estudo (generalização por tempo) foi realizado com uma amostra de 204 participantes proveniente da amostra do segundo estudo, e foi realizado quatro meses depois. Os resultados apresentaram algumas mudanças significativas na dimensão cognitiva e metacognitiva, o que permite concluir que a inteligência cultural é uma capacidade maleável e que varia consoante a exposição cultural, treino, socialização ou outras experiências (Earley \& Ang, 2003). O quarto estudo (generalização por países) foi realizado através da comparação dos resultados obtidos no estudo dois (amostra de 447 participantes de Singapura) e uma amostra de 337 participantes provenientes dos Estados Unidos, revelando igualmente a estrutura de quatro fatores entre os dois países.

Apesar da investigação empírica acerca da inteligência cultural ser relativamente recente, os resultados iniciais são significativos e promissores (Dyne, Ang, \& Nielsen, 2007). Até agora, Ang, Dyne, e Koh (2007) e Ng, Dyne e Ang (2009a, 2009b) evidenciaram que os indivíduos que possuem uma inteligência cultural mais elevada são mais eficientes na tomada de decisões em situações interculturais e têm uma maior probabilidade de se adaptar a situações caracterizadas pela diversidade cultural. Quanto mais elevada for a dimensão metacognitiva da inteligência cultural mais elevada será a capacidade de atuação, e quanto mais elevada a dimensão comportamental melhor será o desempenho em cenários de diversidade cultural. Outros estudos têm sido realizados, nomeadamente relacionando a inteligência cultural com a seleção e treino de expatriados (Ng et al., 2009a), a performance dos expatriados (Ang et al., 2007; Elenkov \& Manev, 2009; Gertsen \& Sodeberg, 2010; Lee, 2010), a liderança global (House, Javidan, Gupta, Dorfman, \& Hanges, 2004; Ng et al., 2009b), o impacto da exposição cultural na inteligência (Crowne, 2008), a adaptabilidade social (Soltani \& Keyvanara, 2013), o nível de inteligência cultural dos professores (Petrović, 2011) e o ajustamento de expatriados (Ang et al., 2007; Bhaskar-Shrinivas, Shaffer, \& Luk, 2005; Ramalu, Wei, \& Rose, 2011).
A inteligência cultural assume-se como um importante construto que permite captar importantes aspetos da estratégia, da motivação, do desempenho e da adaptação eficaz em diversos contextos culturais, tornando-se assim uma potencial ferramenta de diagnóstico do sucesso intercultural (Fischer, 2011). Compreender a natureza e o impacto da inteligência cultural poderá ter importantes implicações no desempenho de indivíduos, equipas ou organizações em ambientes multiculturais (Ng \& Earley, 2006), porque indivíduos com altos níveis de inteligência cultural estão mais predispostos para uma adaptação a ambientes desconhecidos mais eficaz, apresentam maiores capacidades de comunicação verbal e não verbal, identificando facilmente “o que é ou não culturalmente aceitável” (Rego \& Cunha, 2009, p. 59). A inteligência cultural, tornou-se uma aptidão e competência fundamental num mundo social em constante mobilidade, e a escala de inteligência cultural, uma medida imprescindível para compreender os processos de integração e adaptabilidade social.

Dada a atualidade e pertinência do conceito de inteligência cultural e da sua escala, como medida para compreender os processos de integração, o objetivo deste estudo consistiu em contribuir para a adaptação da escala de inteligência cultural para a população Portuguesa.

\section{Método}

\section{Amostra}

A amostra do presente estudo foi recolhida por acessibilidade e foram considerados como critérios de inclusão indivíduos maiores de 18 anos e de nacionalidade portuguesa. Foram considerados apenas os questionários que foram devolvidos, corretamente preenchidos. É uma amostra composta por 275 indivíduos de nacionalidade portuguesa, sendo $32 \%$ do género masculino $(N=88)$ e $66,5 \%$ do género feminino $(N=183)$ sendo que $1,5 \%(N=4)$ não responderam a esta questão. As idades encontram-se compreendidas entre os 18 e os 72 anos $(M=29,3 ; D P$ $=12,9)$. Em relação ao estado civil, cerca de $70,5 \%$ são solteiros, $18,2 \%$ estão divorciados ou separados, $9,8 \%$ são casados ou vivem em união de facto e $1,5 \%$ dos respondentes é viúvo. Cerca de $70,2 \%$ dos respondentes afirma ter contacto com indivíduos de outras nacionalidades no local de trabalho e 79,6\% com amigos ou familiares de outras nacionalidades.

Trata-se de uma amostra heterogénea no tocante à sua naturalidade, nível educacional e áreas de formação. Os participantes são na sua maioria pertencentes às regiões do Alentejo e Algarve (67,3\%), seguidos pela área metropolitana de Lisboa $(13,1 \%)$ e pela região das Beiras, Estremadura e Ribatejo ( $8,4 \%$ ). Os restantes participantes pertencem às regiões do Minho, Douro e Trás-os-Montes, das Ilhas (Açores e Madeira) e da área metropolitana do Porto. No que diz respeito ao nível educacional 59,3\% possui o Ensino Secundário e 30,9\% possui Licenciatura $/ 1^{\circ}$ Ciclo, a restante percentagem encontra-se distribuída pelo Ensino Básico, Mestrado $/ 2^{\circ} \mathrm{Ciclo}$, Doutoramento $/ 3^{\circ} \mathrm{Ciclo}$ 
Sousa, C., Gonçalves, G., Reis, M. \& Santos, J. V. (2015). Evidências Métricas da Adaptação da Escala de Inteligência Cultural numa Amostra Portuguesa.

e Pós-graduação. Em relação à ocupação profissional, que foi categorizada de acordo com a classificação de profissões do INE (Instituto Nacional de Estatística), a maioria dos participantes são estudantes (46,9\%), seguidos de especialistas das actividades intelectuais e científicas $(17,8 \%)$, de técnicos e profissionais de nível intermédio $(9,8 \%)$, de trabalhadores não qualificados $(6,5 \%)$ e de pessoal administrativo (3,3\%). Os restantes são trabalhadores qualificados da agricultura, pesca e florestas $(1,1 \%)$, representantes do poder legislativo, executivo, dirigentes, diretores e gestores executivos $(1,1 \%)$, trabalhadores dos serviços pessoais, de proteção, segurança e vendedores $(0,4 \%)$, trabalhadores qualificados da indústria, construção e artífices $(0,4 \%)$, reformados $(0,4 \%)$ e desempregados $(1,8 \%)$. Cerca de $10,5 \%(N=29)$ dos participantes não responderam a esta questão.

\section{Instrumento}

O instrumento deste estudo foi o The Cultural Intelligence Scale (CQS) originalmente desenvolvido por Dyne et al., (2008) em língua inglesa e aplicado em amostras de estudantes. É constituído por 20 itens cotados numa escala crescente de 1 (Discordo Totalmente) a 7 (Concordo Totalmente). É um instrumento multidimensional, que engloba quatro dimensões de "inteligência": a metacognitiva, a cognitiva, a motivacional e a comportamental. Nos estudos originais, a dimensão metacognitiva apresentou um alfa de Cronbach que variou entre 0,71 e 0,78, na dimensão cognitiva o alfa variou entre 0,81 e 0,85 , na dimensão motivacional o alfa variou entre 0,75 e 0,80 e, por último, a dimensão comportamental apresentou uma alfa de Cronbach que variou entre 0,81 e 0,84 .

Além da escala, acrescentaram-se ainda itens relativos a características biográficas (idade, naturalidade, estado civil e nível educacional) e questões relacionadas com o tipo de contacto mantido com indivíduos de outras nacionalidades, quer na vida profissional, quer na vida pessoal, de forma a permitir caracterizar a amostra.

A validade aparente foi suportada por um processo de tradução-retradução. Inicialmente a escala foi traduzida do inglês para o português, por dois tradutores bilingues, de modo independente. Posteriormente, as duas versões foram retraduzidas para o inglês por outros dois especialistas bilingues, igualmente de modo independente. As traduções obtidas foram comparadas com a versão original e ajustadas para a versão final por psicólogos especialistas na temática. Para testar a tradução, solicitou-se a 15 participantes (pré-teste) que respondessem à versão portuguesa, com vista a corrigir possíveis problemas de compreensão. Deste pré-teste, o alfa de Cronbach foi superior a 0,80 e não foram detetados problemas de interpretação. Estes participantes não foram incluídos na amostra final.

\section{Procedimento}

A recolha da informação foi realizada em diversas situações, nomeadamente em turmas de alunos universitários, em empresas públicas e privadas, em cafés e em bibliotecas. Após a autorização da Comissão Científica (entidade responsável que regula os procedimentos e garantias éticas das investigações) e garantia dos critérios éticos (e.g. informação acerca da liberdade de participar e o anonimato) foi pedido aos participantes que respondessem à escala. O tempo de preenchimento foi de aproximadamente 12 minutos.

\section{Métodos de Análise}

Os dados recolhidos foram tratados estatisticamente no programa SPSS (versão 20.0) e no programa SPSS AMOS (versão 20.0) e a significância foi assumida ao nível de 0,05. As propriedades psicométricas da escala de Inteligência Cultural foram avaliadas através da análise fatorial exploratória, análise fatorial confirmatória e consistência interna. De modo a poder analisar a validade cruzada, a amostra foi divida aleatoriamente em duas partes, 137 participantes para a análise exploratória e 138 participantes para a análise confirmatória afim de realizar análises fatoriais exploratória e confirmatória (Worthington \& Whittaker, 2006). De acordo com Floyd e Widaman (1995) a validade cruzada permite analisar se a estrutura identificada se repete quando investigada numa segunda amostra, tanto para soluções fatoriais exploratórias como para soluções fatoriais confirmatórias, sendo necessário dividir a amostra em dois grupos aleatórios. Para a verificação da validade de construto, a escala de Inteligência Cultural foi submetida a uma análise fatorial exploratória validada pelo critério KMO e com extração dos fatores pelo método das componentes principais seguida de uma rotação oblíqua Promax. Para a análise confirmatória, as matrizes de variância-covariância foram consideradas como entrada, adotando-se o estimador de máxima verossimilhança (ML), que assume a normalidade dos dados, uma vez que é um estimador robusto quando este pressuposto não é cumprido. Foram considerados os seguintes indicadores de ajuste: $\mathrm{O} X^{2}$ representa um teste à significância da função de discrepância minimizada durante o ajustamento do modelo e quanto menor for o seu valor, melhor será o ajustamento (Maroco, 2010). O CMIN/DF complementa a informação sobre o $X^{2}$, ou seja, é a razão entre $X^{2} / g l$. O $X^{2} /$ $g l$ corresponde à probabilidade de ajustamento dos dados ao modelo teórico devendo os seus valores variar entre $2 \mathrm{e}$ 5. O Comparative Fit Index (CFI) pode variar entre 0 e 1, considerando-se que quanto mais próximo de 1 melhor o ajustamento, admitindo-se valores próximos ou superiores a 0,90 para indicação de ajustamento adequado. O Non-normed Fit Index (NNFI) mede o ajustamento relativo do modelo através da comparação da não centralidade de acordo com os graus de liberdade e varia entre 0 e 1 , admitindo-se 0,90 como um valor de bom ajustamento (Bentler \& Bonnet, 1980). A Root-Mean Square Error of Aproximation (RMSEA) caracteriza-se por um intervalo de confiança de $90 \%$ (IC90\%), sendo que o seu valor ideal se situa entre 0,05 e 0,08 , aceitando-se valores até 0,10 . A Standardized Root-Mean Square Residual (SRMR) corresponde à medida da média dos resíduos normalizados e um 
Tabela 1

Componentes Principais Extraídos da Escala de Inteligência Cultural (comunalidades e pesos fatoriais)

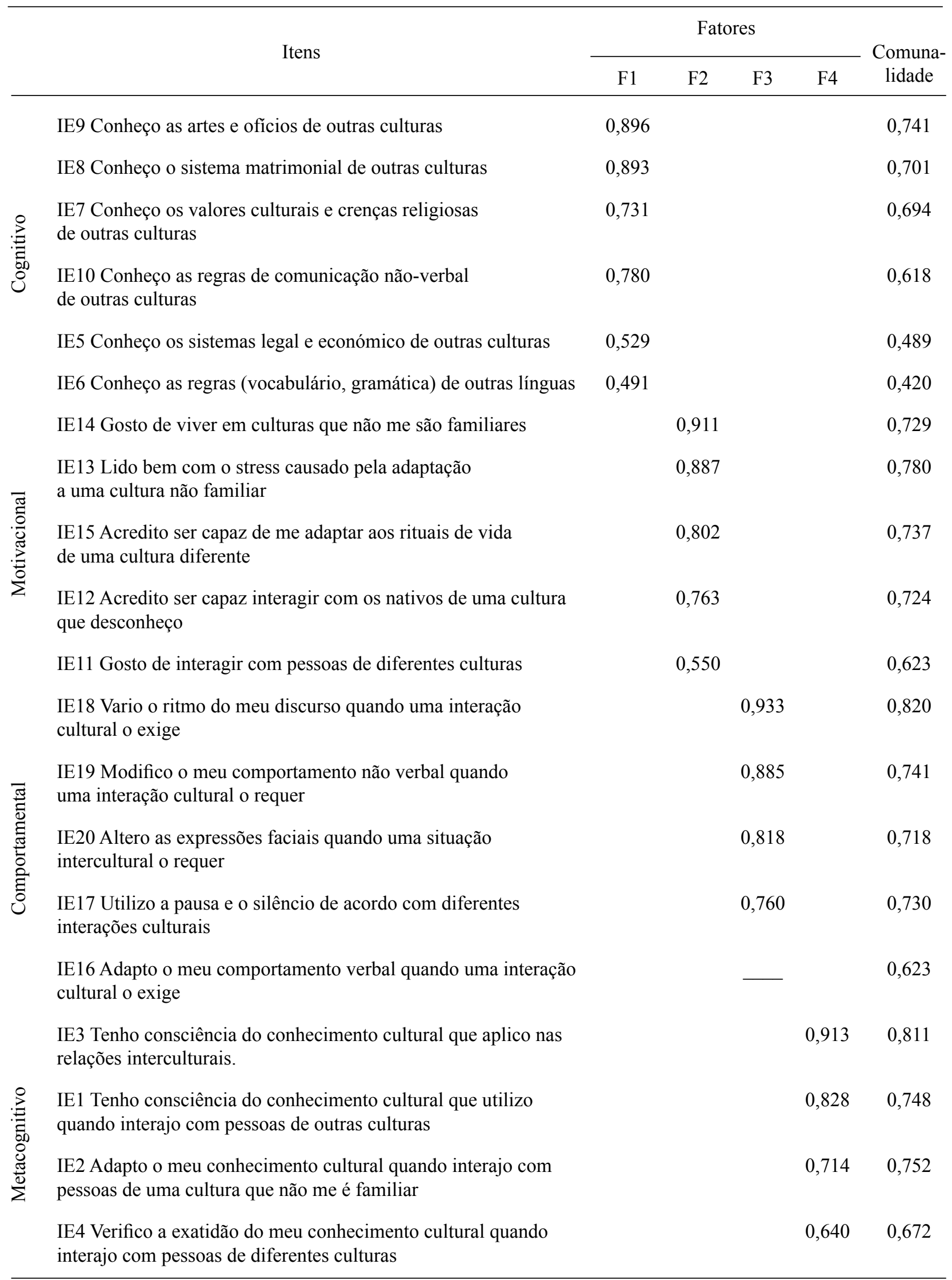

Nota. Método de extração: Maximum Likelihood. Método de Rotação: Promax with Kaiser Normalization. 
Sousa, C., Gonçalves, G., Reis, M. \& Santos, J. V. (2015). Evidências Métricas da Adaptação da Escala de Inteligência Cultural numa Amostra Portuguesa.

ajustamento adequado do modelo é indicado por valores inferiores a 0,05 (Hu \& Bentler, 1999). A consistência interna da escala foi avaliada por meio do coeficiente alfa de Cronbach, que pode variar numa escala de 0 a 1 , admitindo-se como aceitável valores a partir de 0,70 (Nunnally, 1978, citado por Maroco \& Garcia-Marques, 2006).

\section{Resultados}

\section{Análise Fatorial Exploratória}

Com o objetivo de perceber a estrutura da escala realizou-se uma análise exploratória. O índice $\mathrm{KMO}$ apresentou um valor de 0,885 , verificando-se ainda a existência de correlação entre os itens em estudo (teste de esfericidade de Bartlett=1858,008; $g l=190 ; p=0,001)$. A análise das componentes principais, considerando o critério proposto pelo software estatístico SPSS de eigenvalues superiores a 1 para a determinação dos fatores a reter, com recurso à rotação promax permitiu observar 4 fatores, os quais explicam 71,38 \% da variância dos resultados obtidos. $\mathrm{Na}$ Tabela 1 é possível observar os itens distribuídos pelos 4 fatores, coincidente com a versão de Dyne et al. (2008), assim como o seu peso.

O Scree Plot (Figura 1) mostra, que o primeiro fator é constituído pelos itens IE5, IE6, IE7, IE8, IE9 e IE10 que explicam 43,5\% da variância dos resultados (eigenvalue de 8,7). O segundo fator (itens IE11, IE12, IE13, IE14 e IE15) explica $11,63 \%$ da variância dos resultados (eigenvalue de 2,327). O terceiro fator é constituído pelos itens IE16, IE17, IE18, IE19 e IE20 e explica 9,47\% da variância dos resultados (eigenvalue de 1,894). O quarto, e último fator, é constituído pelos itens IE1, IE2, IE3 e IE4 e explica 6,78\% da variância dos resultados (eigenvalue de 1,356). Todas as comunalidades são elevadas (superiores a 0,55 ), com exceção dos itens IE5 e IE6, que apresentam valores de comunalidade de 0,529 e 0,491 respetivamente. A rotação promax permitiu observar uma saturação do IE16 em três fatores $(\mathrm{F} 2 ; \mathrm{F} 3 ; \mathrm{F} 4)$.

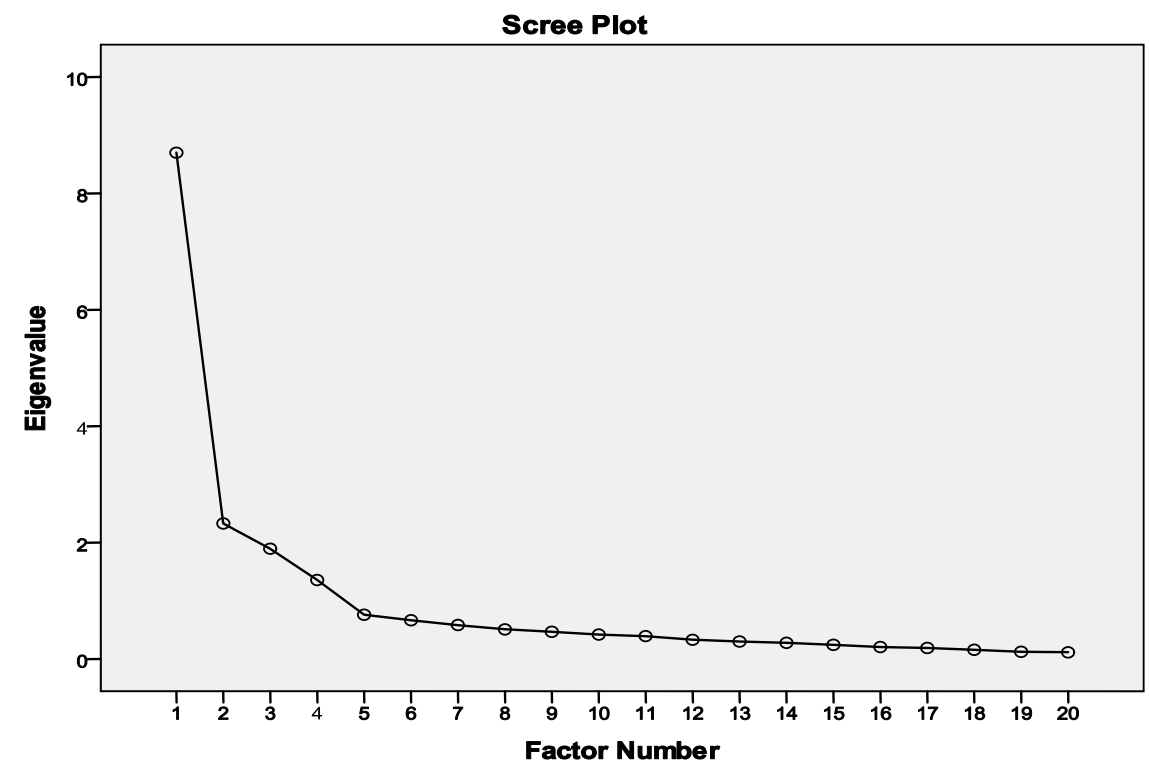

Figura 1. Eigenvalues extraídos da análise fatorial exploratória (Scree Plot).

\section{.Análise Fatorial Confirmatória}

$\mathrm{Na}$ realização da análise fatorial confirmatória testaram-se 3 modelos distintos (Tabela 2). No primeiro modelo, que testa a escala original, os valores de ajustamento obtidos foram: $X^{2}=326,372$ e 164 graus de liberdade, o que se traduz num CMIN/DF de 1,99, sendo este valor um bom indicador (Byrne, 2001).

Os valores de CFI $(0,910)$ e NNFI $(0,896)$ são próximos do valor 1 , o que revela um bom ajustamento (Bentler, 1990; Byrne, 2001; Maroco, 2011). O valor de RMSEA $(0,085)$, embora exceda os 0,06 , não poderá ser considerado um ajuste medíocre pois é inferior a $0,10(\mathrm{Hu} \&$ Bentler, 1999; Ullman, 2006) e o valor de SRMR $(0,07)$, embora superior a 0,05 , é aceitável e próximo do valor obtido pelos autores $(0,06)$.
Com o objetivo de definir um modelo mais ajustado testamos um segundo modelo. Neste modelo foram retirados os itens IE5 e IE6 por apresentarem valores de comunalidade baixos. Neste sentido, testou-se uma solução fatorial de quatro fatores, mas sem os itens referidos anteriormente, ou seja, com 18 itens. Os resultados obtidos foram $X^{2}=260,141$ e 129 graus de liberdade, um CMIN/ $D F$ de 2,01 , os valores de CFI $(0,923)$ e NNFI $(0,909)$ indicam um bom ajustamento já que são valores próximos da unidade. $O$ valor de RMSEA $(0,086)$ é inferior a 0,10 e o valor de SRMR $(0,06)$ é aceitável e igual ao obtido por Dyne et al. (2008).

No terceiro modelo com 4 fatores e 19 itens, foi retirado o IE16 por apresentar valores de saturação em três fatores. Os resultados obtidos nesta testagem foram $X^{2}=284,389 \mathrm{e}$ 
146 graus de liberdade e um CMIN/DF de 1,94. Os valores de CFI $(0,918)$ e NNFI $(0,903)$ são superiores a 0,90 , o que indica um bom ajustamento (Bentler, 1990; Byrne, 2001;
Maroco, 2011), o valor de RMSEA $(0,083)$ e de SRMR $(0,079)$ representam um ajustamento mais fraco. O modelo replica os quatro fatores da escala original, verificando-se a sua multidimensionalidade.

Tabela 2

Resultados dos Estudos Originais de Dyne et al. (2008) e da Adaptação à População Portuguesa

\begin{tabular}{|c|c|c|c|c|c|c|c|c|c|c|}
\hline & & & $n$ & $X^{2}$ & $g l$ & $\mathrm{CMIN} / D F$ & CFI & NNFI & RMSEA & SRMR \\
\hline \multirow{4}{*}{ 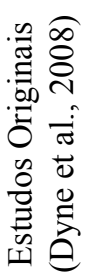 } & Validação da Escala & Estudo 1 & 576 & 822,26 & 164 & - & 0,92 & 0,91 & 0,08 & 0,06 \\
\hline & Generalização por amostras & Estudo 2 & 447 & 381,28 & 164 & - & 0,96 & 0,96 & 0,05 & 0,04 \\
\hline & Generalização por tempo & Estudo 3 & 204 & 981,8 & 692 & - & 0,95 & 0,94 & 0,04 & 0,06 \\
\hline & Generalização por países & Estudo 4 & 337 & 723,23 & 328 & - & 0,97 & 0,96 & 0,05 & 0,05 \\
\hline \multirow{3}{*}{ 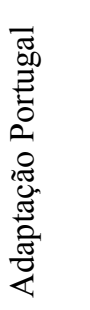 } & $\begin{array}{l}4 \text { Fatores } \\
\text { (20 itens) }\end{array}$ & Modelo 1 & 138 & 326,37 & 164 & 1,99 & 0,91 & 0,89 & 0,08 & 0,07 \\
\hline & $\begin{array}{l}4 \text { Fatores } \\
\text { (18 itens) }\end{array}$ & Modelo 2 & 138 & 260,14 & 129 & 2,01 & 0,92 & 0,90 & 0,08 & 0,06 \\
\hline & $\begin{array}{l}4 \text { Fatores } \\
\text { (19 itens) }\end{array}$ & Modelo 3 & 138 & 284,38 & 146 & 1,94 & 0,92 & 0,90 & 0,08 & 0,07 \\
\hline
\end{tabular}

Nota. CFI, comparative fit index; NNFI, non-normed fit index; RMSEA, root mean square error of approximation; SRMR, standardize root mean square residual.

\section{Consistência Interna}

A consistência interna da escala apresenta um alfa de Cronbach (estratificado) de 0,930. De acordo com Cronbach, Shonenman e McKie (1965, citados por Maroco \& Garcia-Marques, 2006) este alfa destina-se a instrumentos cujos itens podem ser agrupados em fatores de acordo com o seu conteúdo. Relativamente à consistência interna dos fatores, o primeiro fator apresenta um alfa de 0,88 ; o segundo fator um alfa de 0,86 ; o terceiro fator um alfa de 0,88 ; o quarto e último fator apresenta um alfa de 0,89 , valores semelhantes aos observados nos quatro estudos realizados por Dyne et al., (2008) de suporte à construção e validação da escala (Tabela 3 ).

Tabela 3

Valores de Consistência Interna ( $\alpha$ ) de Dyne et al. (2008) e da Adaptação à População Portuguesa

$\alpha$

MetaCognitivo Cognitivo Motivacional Comportamental

\begin{tabular}{|c|c|c|c|c|c|c|}
\hline \multirow{4}{*}{ 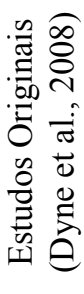 } & Validação da Escala & Estudo 1 & 0,71 & 0,85 & 0,75 & 0,83 \\
\hline & Generalização por amostras & Estudo 2 & 0,77 & 0,84 & 0,77 & 0,84 \\
\hline & Generalização por tempo & Estudo 3 & 0,72 & 0,51 & 0,67 & 0,63 \\
\hline & Generalização por países & Estudo 4 & 0,78 & 0,81 & 0,80 & 0,81 \\
\hline \multirow{2}{*}{ 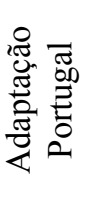 } & $\begin{array}{l}4 \text { Fatores } \\
(20 \text { itens) }\end{array}$ & Modelo 1 & 0,88 & 0,86 & 0,88 & 0,89 \\
\hline & $\begin{array}{l}4 \text { Fatores } \\
19 \text { itens }\end{array}$ & Modelo 3 & 0,88 & 0,86 & 0,88 & 0,90 \\
\hline
\end{tabular}


Sousa, C., Gonçalves, G., Reis, M. \& Santos, J. V. (2015). Evidências Métricas da Adaptação da Escala de Inteligência Cultural numa Amostra Portuguesa.

\section{Discussão e Conclusão}

A Inteligência Cultural é uma capacidade fundamental no mundo laboral. Caracterizada como a capacidade de adaptação e ajustamento à multiculturalidade, esta difere dos restantes tipos de inteligência porque se foca especificamente nas interações caracterizadas pela diversidade cultural. Devido à importância crescente deste construto face à globalização das organizações, o objetivo deste estudo foi contribuir para a adaptação para a população Portuguesa, da escala de inteligência cultural desenvolvida por Dyne et al. (2008). Esta escala, composta por 20 itens, é uma escala multidimensional de quatro dimensões. As dimensões metacognitiva e cognitiva, estão associadas ao julgamento cultural, ao conhecimento de normas, hábitos, valores culturais, à tomada de decisões e ao desempenho. A dimensão motivacional é preditora do ajustamento a ambientes interculturais (redução dos níveis de stress, capacidade para ultrapassar obstáculos, aumento da confiança), e a dimensão comportamental está associada ao comportamento adotado para lidar com situações culturais não familiares, ou seja, para comunicar verbal e não verbalmente os comportamentos adequados à situação.

Com uma amostra de 275 participantes, a versão portuguesa da escala mostrou ser acessível a vários níveis educacionais, não levantando quaisquer problemas de interpretação ou compreensão. Em relação à distribuição por regiões, esta poderia ser mais equilibrada, (já que a maioria dos respondentes $[67,3 \%$ ] pertence às regiões do Alentejo e Algarve), para detetar possíveis dificuldades em relação aos dialetos e regionalismos da língua. Por outro lado, a exposição destas regiões a culturas estrangeiras é mais frequente do que em outras regiões do país, pelo que se torna importante avaliar a escala em regiões com menos contacto.

Para a realização da análise exploratória e confirmatória, a amostra total de 275 participantes foi aleatoriamente dividida em duas amostras $(N=137$ e $N=138$, respetivamente). A análise exploratória apresentou bons valores de validade e boas propriedades métricas semelhantes ao estudo original, revelando igualmente a sua multidimensionalidade já que identificou as quatro dimensões da inteligência cultural (metacognitiva, cognitiva, motivacional e comportamental) propostas por Dyne et al. (2008). Desta análise exploratória, os itens IE5 e IE6 obtiveram valores de comunalidade baixos e o item 16 apresentou valores de saturação em três fatores. Os modelos testados pela análise confirmatória tiveram em consideração os aspetos acima mencionados e o modelo original, resultando em três modelos. Apesar de alguns indicadores apresentarem valores mais fracos (RMSEA e SRMR), são valores que se aproximam dos resultados obtidos pelos autores na sua validação da escala. A consistência interna da escala e das quatro dimensões, calculada através do índice alfa de Cronbach, apresentou valores que se aproximam do estudo original, variando entre 0,71 e 0,89 , e que representam um bom índice de fiabilidade.
Sendo a inteligência cultural uma capacidade maleável, associada a um comportamento adaptativo em novos contextos e que varia consoante a exposição cultural, treino, socialização ou outras experiências (Earley \& Ang, 2003), poderia ser realizado um teste-reteste (tal como realizado nos estudo de Dyne et al. (2008). Uma estratégia seria aplicar a escala de inteligência cultural no início do ano letivo, a uma turma de alunos, e após alguns meses voltar a aplicá-la, verificando se existem alterações significativas nalguma dimensão da inteligência cultural. Ou, replicando o estudo de Petrović (2011), aplicar a escala de inteligência cultural a professores, que lidam diariamente com alunos de várias culturas, analisando o seu nível de inteligência cultural. Em investigações futuras poderia ser realizado um estudo experimental, introduzindo alterações controladas no comportamento (e.g. situações de conflito entre grupos multiculturais), e verificar se os resultados diferem. Com uma amostra maior, poderia também ser realizada uma análise fatorial confirmatória numa sub-amostra e posteriormente uma análise multigrupos com o objetivo de verificar a estabilidade do modelo. A validade preditiva pode constituir um objetivo a considerar noutros estudos. Por exemplo, recorrendo a indivíduos selecionados para processos de expatriação, para cargos de líderes globais ou para a realização de negociações interculturais. Para a validade do instrumento por critério externo, poderia correlacionar-se a escala de inteligência cultural com o questionário de personalidade multicultural de Van der Zee e Van Oudenhoven (2000), que permite identificar disposições específicas dos indivíduos na adaptação cultural, suas competências e sua eficácia multiculturais.

Ser culturalmente inteligente é uma característica cada vez mais importante nas organizações de hoje. A escala de inteligência cultural será uma mais valia para as organizações, já que irá permitir determinar quais os indivíduos mais aptos para lidar com diferentes culturas, aumentando assim a taxa de sucesso dos processos de expatriação e internacionalização, e consequentemente na gestão dos recursos humanos e nas dinâmicas sociais (e.g. Clipa \& Clipa, 2009; Fajardo, 2007).

\section{Referências}

Ang, S., Dyne, L., \& Koh, C. (2006). Personality correlates of the four-fator model of cultural intelligence. Group and Organization Management, 31, 100-123. doi:10.1177/1059601105275267

Ang, S., Dyne, L., \& Koh, C. (2007). Cultural intelligence: Its measurement and effects on cultural judgment and decision making, cultural adaptation, and task performance. Management and Organization Review, 3, 335-371. doi:10.1111/ j.1740-8784.2007.00082.x

Bentler, P. M. (1990). Comparative fit indexes in structural models. Psychological Bulletin, 107(2), 238-246.

Bentler, P. M., \& Bonett, D. G. (1980). Significance tests and goodness of fit in the analysis of covariance structures. Psychological Bulletin, 88, 588-606. 
Bhaskar-Shrinivas, P., Shaffer, M. A., \& Luk, D. M. (2005). Input-based and time-based models of international adjustment: Meta-analytic evidence and theoretical extensions. Academy of Management Journal, 482, 25-281. doi:10.2307/20159655

Byrne, B. (2001). Structural equation modeling with AMOS: Basic concepts, applications and programming. Mahwah, NJ: Lawrence Erlbaum.

Cantor, N., \& Kihlstrom, J. (1985). Social intelligence: The cognitive basis of personality. Review of Personality and Social Psychology, 6, 15-33.

Clipa, F., \& Clipa, R. (2009). Cultural diversity and human resource management in multinational companies. CES Working Papers, 1(1), 10-16. Retrieved from http://www.ceswp.uaic. ro/articles/CESWP2009_I1_CLI.pdf

Crowne, K. (2008). What leads to cultural intelligence? Business Horizons, 51, 391-399. doi:10.1016/j.bushor.2008.03.010

Dyne, L., Ang, S. \& Koh, C. (2008). Development and validation of the CQS. In S. Ang \& L. Dyne (Eds.), Handbook of cultural intelligence. Theory, measurement and applications (pp. 16-38). New York: Sharpe.

Dyne, L., Ang, S., \& Livermore, D. (2010). Cultural intelligence: A pathway for leading in a rapidly globalizing world. In $\mathrm{K}$. Hannum, B. B. McFeeters, \& L. Booysen (Eds.), Leading across differences (pp. 131-138). San Francisco, CA: Pfeiffer.

Dyne, L., Ang, S., \& Nielsen, T. M. (2007). Cultural intelligence. In S. Clegg \& J. Bailey (Eds.), International encyclopedia of organization studies (Vol. 1, pp. 345-350). Thousand Oaks, CA: Sage.

Earley, P. C. (2002). Redefining interactions across cultures and organizations: Moving forward with cultural intelligence. Research in Organizational Behavior, 24, 271-299. doi:10.1016/ S0191-3085(02)24008-3

Earley, P. C., \& Ang, S. (2003). Cultural intelligence: Individual Interactions across Cultures. Palo Alto, CA: Stanford University Press.

Earley, P. C., \& Mosakowski, E. (2004, October). Cultural intelligence. Harvard Business Review, 139-153. Retrieved from http://hbr.org/2004/10/cultural-intelligence/ar/1

Elenkov, D. S., \& Manev, I. M. (2009). Senior expatriate leadership's effects on innovation and the role of cultural intelligence. Journal of World Business, 44, 357-369. doi:10.1016/j.jwb.2008.11.001

Fajardo, V. (2007). Multiculturalidad:¿Ventaja u obstáculo de las organizaciones? Signo y Pensamiento, 26(51), 109-117. Recuperado de http://revistas.javeriana.edu.co/index.php/ signoypensamiento/article/viewFile/4597/3565

Fan, Y. (2000). A classification of Chinese culture. Cross Cultural Management, 7(2), 3-10.

Fischer, R. (2011). Cross-cultural training effects on cultural essentialism beliefs and cultural intelligence. International Journal of Intercultural Relations 35, 767-775. doi:10.1016/j. ijintrel.2011.08.005

Floyd, F., \& Widaman, K. (1995). Factor analysis in the development and refinement of clinical assessment instruments. Psychological Assessment, 7, 286-299.

Gardner, H. (1983). The frame of mind: The theory of multiple intelligences. New York: Basic Books.

Gertsen, M. C., \& Soderberg, A. (2010). Expatriate stories about cultural encounters - A narrative approach to cultural learning processes in multinational companies. Scandinavian Journal of Management, 26, 248-257. doi:10.1016/j.scaman.2010.06.003
Goleman, D. (1996). Inteligência emocional. Lisboa, Portugal: Círculo de Leitores.

Goleman, D. (2006). Inteligência social. A nova ciência das relações humanas. Barcelos, Portugal: Círculo de Leitores.

House, R. J., Javidan, M., Gupta, V., Dorfman, P. W., \& Hanges, P. J. (Eds). (2004). Culture, leadership and organizations: The GLOBE study of 62 societies. Thousand Oaks, CA: Sage.

Hu, L., \& Bentler, P. M. (1999). Cutoff criteria for fit indexes in covariance structure analysis: Conventional criteria versus new alternatives. Structural Equation Modeling, 6, 1-55. doi:10.1080/10705519909540118

Lee, L.-Y. (2010). Multiple intelligences and the success of expatriation: The roles of contingency variables. African Journal of Business Management, 4, 3793-3804. Retrieved from http://www.academicjournals.org/AJBM

Maroco, J. (2010). Análise de equações estruturais. Fundamentos teóricos, software e aplicações. Pêro Pinheiro, Portugal: ReportNumber.

Maroco, J. (2011). Análise estatística com o SPSS Statistics (5. ed.). Pêro Pinheiro, Portugal: ReportNumber.

Maroco, J., \& Garcia-Marques, T. (2006). Qual a fiabilidade do alfa de Cronbach? Questões antigas e soluções modernas. Laboratório de Psicologia, 4(1), 65-90.

Ng, K. Y., \& Earley, P. C. (2006). Culture+intelligence: Old constructs, new frontiers. Group and Organization Management, 31, 4-19. doi:10.1177/1059601105275251

Ng, K. Y., Dyne, L., \& Ang, S. (2009a). Beyond international experience: The strategic role of cultural intelligence for executive selection in HRM. In P. R. Sparrow (Ed.), Handbook of international human resource management (pp. 97-113). London: John Wiley \& Sons.

Ng, K. Y., Dyne, L., \& Ang, S. (2009b). Developing global leaders: The role of international experience and cultural intelligence. Advances in Global Leadership, 5, 225-250. doi:10.1108/S1535-1203(2009)0000005013

Petrović, D. S. (2011). How do teachers perceive their cultural intelligence? Procedia Social and Behavioral Sciences, 11, 276-280. doi:10.1016/j.sbspro.2011.01.076

Ramalu, S., Wei, C., \& Rose, R. (2011). The effects of cultural intelligence on cross-cultural adjustment and job performance amongst expatriates in Malasya [Special issue]. International Journal of Business and Social Science, 2(9), 59-71. Retrieved from http://ijbssnet.com/journals/Vol._2_No._9_[Special_Issue_-_May_2011]/9.pdf

Rego, A., \& Cunha, M. P. (2009). Manual de gestão transcultural de Recursos Humanos. Lisboa, Portugal: RH.

Rockstuhl, T., Hong, Y., Ng, K. Y., Ang, S., \& Chiu, C. (2010). The culturally intelligent brain: From detecting to bridging cultural differences. NeuroLeadership Journal, 3, 1-15. Retrieved from http://www.culturalq.com/docs/Rockstuhl_ et_al_2010.pdf

Rose, R., Ramalu, S., Uli, J., \& Kumar, N. (2010). Expatriate performance in international assignment: The role of cultural intelligence as dynamic intercultural competency. International Journal of Business and Management, 5(8), 76-85. Retrieved from www.ccsenet.org/ijbm

Soltani, B., \& Keyvanara, M. (2013). Cultural intelligence and social adaptability: A comparison between Iranian and NonIranian dormitory students of Isfahan University of Medical Sciences. Materia Socio Medica, 25(1), 40-43. doi:10.5455/ msm.2013.25.40-43 
Sousa, C., Gonçalves, G., Reis, M. \& Santos, J. V. (2015). Evidências Métricas da Adaptação da Escala de Inteligência Cultural numa Amostra Portuguesa.

Sternberg, R. (1988). The triarchic mind: A new theory of human intelligence. New York: Viking.

Sternberg, R. (2000). Handbook of intelligence. New York: Cambridge University Press.

Sternberg, R. (2004). Culture and intelligence. American Psychologist, 59(5), 325-338. doi:10.1037/0003-066X.59.5.325

Sternberg, R. J., \& Detterman, D. K. (1986). What is intelligence? Contemporary viewpoints on its nature and definition. Norwood, NJ: Ablex.

Ullman, J. B. (2006). Structural equation modeling: Reviewing the basics and moving forward. Journal of Personality Assessment, 87(1), 35-50. doi:10.1207/s15327752jpa8701_03

Van der Zee, K. I., \& Van Oudenhoven, J. P. (2000). The Multicultural Personality Questionnaire: A multidimensional instrument of multicultural effectiveness. European Journal of Personality, 14, 291-309. doi:10.1002/10990984(200007/08)14:4<291::AID-PER377>3.0.CO;2-6

Worthington, R. L., \& Whittaker, T. A. (2006). Scale development research: A content analysis and recommendations for best practices. The Counseling Psychologist, 34, 806-838. doi:10.1177/0011000006288127 